\title{
Serum Creatine Kinase Levels are not Associated with an Increased Need for Continuous Renal Replacement Therapy in Patients with Acute Kidney Injury Following Rhabdomyolysis
}

\author{
Luniu Xiao \\ Huazhonng University of Science and Technology \\ Xiao Ran \\ Huazhonng University of Science and Technology \\ Yanxia Zhong \\ Huazhonng University of Science and Technology \\ Yue Le \\ Huazhonng University of Science and Technology \\ Shusheng Li ( $\nabla$ shushengli16@sina.com ) \\ Huazhonng University of Science and Technology
}

\section{Research Article}

Keywords: Rhabdomyolysis, Acute Kidney Injure, Continuous Renal Replacement Therapy, Creatine Kinase

Posted Date: November 17th, 2021

DOI: https://doi.org/10.21203/rs.3.rs-1049043/v1

License: (c) (1) This work is licensed under a Creative Commons Attribution 4.0 International License.

Read Full License 


\section{Abstract}

\section{Backgroud}

Rhabdomyolysis is a syndrome caused by the breakdown and necrosis of skeletal muscle tissues. As a result, there is leakage of various intercellular myocyte contents into the circulating blood stream. Severe rhabdomyolysis can lead to acute kidney injury (AKI) and cause potentially permanent kidney damage. Previous studies have reported benefit from continuous renal replacement therapy (CRRT) for rhabdomyolysis-associated AKI. For patients with AKI, the termination of CRRT often depends on the patient's renal functions. Here, we asked whether serum creatine kinase (CK) levels should be considered for CRRT termination in patients with AKI following rhabdomyolysis.

Methods

We compared different CK levels in patients after CRRT termination and we observed the correlation between CK levels and clinical outcomes. For a retrospective study, we collected 86 cases with confirmed rhabdomyolysis-associated AKI, who had received CRRT from January 1st of 2012 to December 31th of 2020 in Tongji Hospital. Patients' renal functions were assessed within 24 hours of intermission, and patients with urine output $\geq 1,000 \mathrm{~mL}$ and serum creatinine $\leq 265 \mathrm{umol} / \mathrm{L}$ were considered for CRRT termination. Following CRRT termination, patients were divided into a $\mathrm{CK}>5,000 \mathrm{U} / \mathrm{L}$ group (experimental group) and a $\mathrm{CK}<5,000 \mathrm{U} / \mathrm{L}$ group (control group). The outcomes, such as in-hospital mortality and inhospital length of stay, were compared between two groups.

Results

Thirty-three (38.37\%) patients were classified as having CK > 5,000 U/L, while 53 (61.63\%) were categorized as having $\mathrm{CK}<5,000 \mathrm{U} / \mathrm{L}$. The majority of laboratory examinations were comparable between the two groups on admission. The higher CK levels, as well as worse renal functions, predicted the necessity of CRRT continuation for patients. After CRRT termination, the in-hospital mortality $(27.27 \%$ vs 22.64, $p=0.389$ ) and Multiple Organ Dysfunction Syndrome (MODS) incidence (51.52\% vs $49.06 \%, p=$ 0.064 ) were similar between two groups, while the experimental group showed a significantly shorter inhospital length of stay (11.88 \pm 1.469 vs $16.42 \pm 1.290, p=0.026)$ and Intensive Care Unit (ICU) length of stay $(7.545 \pm 0.866$ vs $10.11 \pm 0.793, p=0.038)$.

Conclusion

CRRT termination may be independent of $s$ the CK levels for patients with rhabdomyolysis-associated acute kidney injure, providing their renal functions have recovered to an appropriate level. Prospective clinical trials would be needed to more thoroughly investigate the optimal CK range that could be used as a gauge to prevent recurrence of renal impairments after treatments.

\section{Introduction}


Rhabdomyolysis (RM) describes a syndrome caused by the disintegration of skeletal muscle tissues, leading to the leakage of various intercellular myocyte contents into the blood stream[1]. The etiology of muscle injury in rhabdomyolysis is diverse, including direct trauma, toxins, infections, and strenuous exercise [2]. The clinical symptoms of RM vary significantly, from an asymptomatic increase in serum levels of enzymes released from damaged skeletal muscles to obvious muscle weakness, myalgia, and dark urine[3]. Severe cases are characterized by metabolic syndromes, including volume depletion, electrolyte abnormalities and even acute kidney injury (AKI).

A diagnosis of RM is given when there are severe muscle symptoms with creatine kinase (CK) levels > $1,000 \mathrm{U} / \mathrm{L}$ or greater than five times the normal limit[4]. Serum levels of CK correlate with the severity of RM[5]: Moderate RM is defined as CK levels within 5,000 - 15,000 U/L, and severe cases are above $15,000 \mathrm{U} / \mathrm{L}$. A recently published review concluded that increasing levels of CK, or failure of levels to decline despite therapies, suggest an ongoing muscle injury and development of AKI[3].

AKI is a feared and common complication of RM, occurring in $13 \%-50 \%$ of patients[6], and up to $7 \%$ $10 \%$ of AKI is attributed to RM[7]. In critically ill patients, AKI doubles mortality with a rate of $59 \%$, compared to $22 \%$ in patients without AKI[8]. Although the pathogenesis of RM - induced AKI is not fully elucidated, previous experimental evidence suggests that renal vasoconstriction, direct and ischemic tubule injury, and tubular obstruction are three major mechanisms involved in disease progression[9].

The myoglobin released from the damaged muscles plays a dominant role in the pathogenesis of RM induced AKI[10]. Myoglobin is an iron-containing small protein with a molecular weight of $17.8 \mathrm{kDa}$ and exits at a very low concentration in the serum under physiological conditins[11]. But for a skeletal muscle injury, serum myoglobin levels increase within one hour and return to a normal range within 1 to 6 hours after lesion resolution. Myoglobin is freely filtered by the glomerulus and is absorbed in the proximal tubule by endocytosis[12]. Excess myoglobin released into the circulation will lead to myoglobinuria and renal insufficiency, which brings about renal tubular obstruction and oxidative injury. Persistently increased serum creatinine, as well as a decreased glomerular filtration rate (eGFR), predicts a deterioration of renal functions, resulting in AKI.

Since the molecular weight of myoglobin is relatively large and difficult to remove by conventional low permeable membranes, myoglobin could be eliminated most effectively by continuous renal replacement therapy CRRT, as compared with traditional intermittent dialysis[13]. Previous studies have reported significant benefits for patients with RM-associated AKI who received early CRRT, including reduced incidence of MODS, lower levels of inflammatory factors, and rapid recovery of renal function[14-17].

For patients with AKI, the termination of CRRT often depends on the patient's renal function at intermission. For example, once urine output is above $1,000 \mathrm{~mL}$, and serum creatinine is below 265 umol/L within 24 hours at intermission, CRRT could be considered for suspension [18]. However, in light of RM-associated AKI, the higher level of CK predicts an ongoing muscle injury, as well as a potentially increased risk of renal dysfunction. Whether the CK levels should be also considered for CRRT 
termination is still controversial. Therefore, we performed a retrospective study to compare different CK levels at CRRT termination and identify its impact on clinical prognosis.

\section{Materials And Methods}

\section{Data collection}

For a retrospective study, we collected 86 cases with confirmed RM-associated AKI, who had received CRRT from January 1st of 2012 to December 31th of 2020 in Tongji Hospital Affiliated to Tongji Medical College of Huazhong University of Science and Technology. The diagnostic criteria for RM were serum CK levels of $>1,000 \mathrm{U} / \mathrm{L}$, along with clinical symptoms, such as muscle weakness, myalgia, or darkcolored urine. All patients underwent the urinalysis (dipstick and microscopy) to differentiate between hematuria and myoglobinuria, and positive urine dipstick tests were confirmed with the absence of red blood cells in the urine sediment.

AKI was defined using a modified Risk, Injury, Failure, Loss, End Stage Renal Disease (RIFLE) classification system, based on the eGFR criteria. All patients were stratified as "Risk" if the serum creatinine value was 1.5 times greater than baseline, "Injury" if it was two times greater, and "Failure" if it was three times greater[4]. Traditional treatments of RM-associated AKI focused on classic protective measurements, including early and aggressive fluid resuscitation, alkalinization of urine, and forced diuresis by osmotic agents. The CRRT was applied for patients with at least one of the following criteria: (1) oliguria (urine output $<400 \mathrm{~mL} / \mathrm{D}$ ) or anuria (urine output $<100 \mathrm{~mL} / \mathrm{D}$ ), for more than two days, (2) symptoms of uremia, such as vomiting, apathy, irritability or drowsiness (3) blood $\mathrm{PH}<7.15$, or serum bicarbonate ion less than $15 \mathrm{mmol} / \mathrm{L}$, (4) blood urea nitrogen above $17.8 \mathrm{mmol} / \mathrm{L}$, or serum creatinine $\geq$ $442 \mathrm{umol} / \mathrm{L}$, (5) obvious electrolyte disturbances, such as serum potassium $\geq 6.5 \mathrm{mmol} / \mathrm{L}$ or sodium $\geq$ $160 \mathrm{mmol} / \mathrm{L}$.

\section{Standardized Treatments with CRRT}

All participants underwent an early and vigorous fluid resuscitation for prevention of shock. Sodium bicarbonate therapy was replenished for patients with obvious metabolic acidosis (blood PH $<7.15$ ). CRRT was either continuous venovenous hemodialysis (CVVHD) or continuous venovenous hemodiafiltration (CVVHDF). CVVHD used standard capillary hemofilters (AV 600S; Fresenius Medical Care, Bad Homburg, Germany; blood flow of $150 \mathrm{~mL} / \mathrm{min}$, dialysate exchange rate $1,000 \mathrm{~mL} / \mathrm{min}$ ). CVVHDF also used the above hemofilters (AV 600S; Fresenius Medical Care, Bad Homburg, Germany; blood flow of $180 \mathrm{~mL} / \mathrm{min}$, dialysate exchange rate $1,500 \mathrm{~mL} / \mathrm{min}$ ). The average CRRT period was sixteen hours, and intermission was 24 hours. Ultrafiltration was adjusted according to clinical need. All materials were employed within the limits of intended use.

\section{Study population}


For a retrospective analysis, the demographic characteristics, etiology, Acute Physiology and Chronic Health Evaluation II score (APACHEII), McMahon score, and laboratory examinations for 86 cases with RM-associated AKI were collected from electronic medical records. According to the British Renal Association guidelines for $\mathrm{AKI}$, we chose urine output and serum creatinine at intermission, as indicators to monitor the renal function recovery. Once the urine output reached $>1,000 \mathrm{~mL}$, with a serum creatinine $\leq 265 \mathrm{umol} / \mathrm{L}$, within $24 \mathrm{~h}$ at intermission, the CRRT was terminated.

For patients with confirmed RM-associated AKI, serum CK levels were dynamically monitored through the full course of CRRT therapy, since the CK levels could reflect the disease process, as well as therapeutic effects. Patients were divided into two groups according to the serum CK levels after CRRT termination. The experimental group (CK levels still $>5,000 \mathrm{U} / \mathrm{L}$ after termination), and control group (CK levels $<5,000$ $\mathrm{U} / \mathrm{L}$ after termination). In the history of RM classification, levels of CK have been thought of as a key factor in predicting the risk of kidney injury. Higher CK levels after termination might predict a non-overt sustained RM, despite standard CRRT treatment. Patients with moderate or severe RM (CK levels $>5,000$ $\mathrm{U} / \mathrm{L}$ ) suffered from a potential development of renal failure, which could potentially bring about CRRT. Different clinical prognoses were compared between two groups to explore whether levels of CK should be also considered an indicator for CRRT termination. For patients with severe RM after CRRT and CK levels above 15,000 U/L, a subgroup analysis was conducted to discuss their relationship with outcomes, since these participants still showed a potential increased risk of dialysis by standard RM classification.

\section{Outcome measurements}

The primary clinical outcome of this research was the in-hospital mortality, while the secondary outcomes included in-hospital length of stay, ICU length of stay, length of CRRT period, and MODS incidence. Laboratory examinations, including routine blood test, CK, myoglobin, renal function variables, and inflammatory indicators, were measured for RM-associated AKI on admission, as well as CRRT termination. Correlations between CK and other variables were analyzed after termination.

\section{Statistical analysis}

Continuous variables were presented as mean \pm standard deviation and checked by Student's T-test. Categorical variables were displayed as frequencies and percentages and analyzed by Chi-square (twosided). Linear correlations between different variables were determined by Spearman rank correlation analysis. $\mathrm{P}<0.05$ was set as a statistical significance, and all data management and analyses were performed using Graphpad Prism 6.0.

\section{Results}

\section{Baseline characteristics of patients with RM associated AKI}

From January 1st, 2012 to December 31th, 2020, 83 patients with RM- associated AKI underwent CRRT in our hospital. Demographic characteristics of study participants are presented in Table 1 . The average age 
was 43 years, and approximately half were male. Reasons for RMinclude, in descending order: infections (36.2\%), toxins (26.7\%), strenuous exercise $(15.1 \%)$, heat stroke $(15.1 \%)$, and trauma $(6.9 \%)$. The mean McMahon score was seven, which predicted a sensitivity and specificity risk of CRRT. The APACHEII score for all subjects was within sixteen to twenty-three. After CRRT termination, 33 patients with CK levels above $5,000 \mathrm{U} / \mathrm{L}$ were assigned to an experimental group, and the remaining 53 cases were assigned to a control group.

Table 1. Demographic characteristic of patients with RB associated AKI.

\begin{tabular}{|ll|}
\hline Patient Characteristics & $\begin{array}{l}\mathrm{N} \% \text { or Median } \\
\text { [Range: Min Max] }\end{array}$ \\
\hline Age, mean, y & $43[18,86]$ \\
\hline Sex, male, $\mathrm{n}(\%)$ & $55(63.9)$ \\
\hline Etiology, $\mathrm{n}(\%)$ & \\
\hline Trauma & $6(6.9)$ \\
\hline Toxins & $23(26.7)$ \\
\hline Infections & $31(36.2)$ \\
\hline Strenuous Exercise & $13(15.1)$ \\
\hline Heat Stroke & $13(15.1)$ \\
\hline McMahon Score & $7[5,10]$ \\
\hline APACHEII Score & $19[16,23]$ \\
\hline
\end{tabular}

APACHEII Score: Acute Physiology and Chronic Health Evaluation II Score

\section{Laboratory Markers On Admission}

Baseline laboratory markers for 86 cases were collected on admission (Table 2 and Figure 1). The results of this study showed that leukocytes, neutrophils, lymphocytes, and hemoglobin were comparable between two groups (all $p$-value $>0.05$ ). In addition, CK and myoglobin, which represented a condition of muscle injury, were also similar $(p=0.081, p=0.103)$. The average urine output was below $400 \mathrm{~mL} / \mathrm{d}$, and the serum creatinine was $497.2 \mathrm{umol} / \mathrm{L}$. These two groups displayed an analogous state of renal function and belonged to the "Failure" stage according to RIFLE classification (Urine output: $p=0.098$, Creatinine: $p=0.344$, Urea Nitrogen: $p=0.072$, eGFR: $p=0.183$ ). There was no difference about inflammatory biomarkers, Procalcitonin (PCT) and C-Reaction Protein (CRP), between two groups $(p=$ $0.863, p=0.859)$. 
Table 2

Laboratory examination of patients with RM associated AKI on admission.

\begin{tabular}{|lllll|}
\hline Variables & $\begin{array}{l}\text { All } \\
(\mathbf{n}=\mathbf{8 6})\end{array}$ & $\begin{array}{l}\text { CK }>\mathbf{5 0 0 0} \mathbf{U} / \mathrm{L} \\
(\mathbf{n}=\mathbf{3 3})\end{array}$ & $\begin{array}{l}\text { CK<5000 U/L } \\
(\mathbf{n}=\mathbf{5 3})\end{array}$ & P value \\
\hline Leukocyte $\left(\times 10^{9} / \mathrm{L}\right)$ & $14.38 \pm 1.195$ & $15.08 \pm 1.233$ & $13.94 \pm 1.157$ & 0.520 \\
\hline Neutrophils $\left(\times 10^{9} / \mathrm{L}\right)$ & $8.516 \pm 1.191$ & $8.430 \pm 1.523$ & $8.651 \pm 0.858$ & 0.177 \\
\hline Lymphocyte $\left(\times 10^{9} / \mathrm{L}\right)$ & $1.101 \pm 0.090$ & $1.173 \pm 0.094$ & $1.029 \pm 0.085$ & 0.367 \\
\hline Hemoglobin $(\mathrm{g} / \mathrm{L})$ & $114.9 \pm 5.494$ & $120.4 \pm 6.650$ & $111.4 \pm 4.338$ & 0.239 \\
\hline CK $(\mathrm{U} / \mathrm{L})$ & $11088 \pm 1494$ & $13419 \pm 1546$ & $9566 \pm 1442$ & 0.081 \\
\hline Myoglobin $(\mathrm{ng} / \mathrm{mL})$ & $1169 \pm 32.87$ & $1213 \pm 39.84$ & $1138 \pm 25.89$ & 0.103 \\
\hline Urine Output $(\mathrm{mL} / \mathrm{d})$ & $368.6 \pm 46.13$ & $395.2 \pm 53.87$ & $327.2 \pm 38.39$ & 0.098 \\
\hline Creatinine $(\mathrm{umol} / \mathrm{L})$ & $497.2 \pm 29.54$ & $474.6 \pm 26.65$ & $515.8 \pm 32.43$ & 0.344 \\
\hline Urea Nitrogen $(\mathrm{mmol} / \mathrm{L})$ & $16.89 \pm 1.935$ & $13.76 \pm 2.096$ & $18.85 \pm 1.775$ & 0.072 \\
\hline eGFR (mL/min/L) & $46.27 \pm 7.158$ & $55.01 \pm 8.114$ & $41.26 \pm 6.201$ & 0.183 \\
\hline PCT (ng/mL) & $2.368 \pm 0.492$ & $2.296 \pm 0.561$ & $2.416 \pm 0.423$ & 0.863 \\
\hline CRP (mg/L) & $62.43 \pm 12.69$ & $64.31 \pm 12.53$ & $61.03 \pm 12.85$ & 0.859 \\
\hline CK: Creatine Kinase; eGFR: Glomerular Filtration Rate; PCT: Procalcitonin; CRP: C-Reaction Protein. \\
\hline
\end{tabular}

\section{Variables After Crrt Termination}

After CRRT termination, patients in the two groups showed a similar APACHEIl score, as well as McMahon score $(p=0.742, p=0.355)$. The mean McMahon score was four points for all participants, which predicted a lower potential risk of CRRT again. Another important finding was that the level of myoglobin was significantly higher in the experimental group ( $852.1 \pm 79.14$ vs $579.7 \pm 63.66, p=0.010$ ), except for the obvious higher CK levels. Notably, urine output, serum creatinine, urea nitrogen, and eGFR, reflecting recovery of renal function,, were comparable ( $p$-value $>0.05$ ). All patients reached the goal of urine output of $>1,000 \mathrm{~mL} / \mathrm{D}$, with serum creatinine $\leq 265 \mathrm{umol} / \mathrm{L}$ after CRRT. In addition, the inflammatory response was also similar, and under a controllable condition ( $p$-value >0.05). Table 3 and Figure 2. 
Table 3

Variables of patients with RM associated AKI after CRRT termination.

\begin{tabular}{|c|c|c|c|c|}
\hline Variables & $\begin{array}{l}\text { All } \\
(n=86)\end{array}$ & $\begin{array}{l}C K>5000 \mathrm{U} / \mathrm{L} \\
(\mathrm{n}=33)\end{array}$ & $\begin{array}{l}C K<5000 \mathrm{U} / \mathrm{L} \\
(n=53)\end{array}$ & $P$ value \\
\hline APACHEII Score & $16.55 \pm 0.305$ & $16.34 \pm 0.338$ & $16.72 \pm 0.272$ & 0.742 \\
\hline McMahon Score & $4.68 \pm 0.221$ & $5.17 \pm 0.242$ & $4.22 \pm 0.199$ & 0.355 \\
\hline CK (U/L) & $4495.2 \pm 586.34$ & $11226 \pm 1080$ & $685.4 \pm 92.68$ & $<0.001$ \\
\hline Myoglobin (ng/mL) & $627.3 \pm 71.40$ & $852.1 \pm 79.14$ & $579.7 \pm 63.66$ & 0.010 \\
\hline Urine Output (mL/d) & $1215 \pm 123.3$ & $1200 \pm 145.9$ & $1224 \pm 100.6$ & 0.890 \\
\hline Creatinine (umol/L) & $179.6 \pm 24.02$ & $176.4 \pm 28.58$ & $181.8 \pm 19.46$ & 0.873 \\
\hline Urea Nitrogen (mmol/L) & $11.50 \pm 1.250$ & $10.83 \pm 1.343$ & $11.92 \pm 1.157$ & 0.549 \\
\hline eGFR (mL/min/L) & $59.16 \pm 7.876$ & $58.76 \pm 8.951$ & $59.38 \pm 6.801$ & 0.956 \\
\hline РCT (ng/mL) & $2.491 \pm 0.800$ & $2.853 \pm 0.992$ & $2.293 \pm 0.599$ & 0.610 \\
\hline $\mathrm{CRP}(\mathrm{mg} / \mathrm{L})$ & $42.11 \pm 17.32$ & $51.32 \pm 20.87$ & $35.32 \pm 13.77$ & 0.510 \\
\hline
\end{tabular}

Outcomes Of Subjects With Rm-associated Aki After Crrt Termination

After CRRT termination, we found that the length of CRRT period was significantly longer in patients with $\mathrm{CK}<5,000 \mathrm{U} / \mathrm{L}(7.830 \pm 0.909$ vs $4.879 \pm 0.792, p=0.027)$. Although the in-hospital mortality showed no difference between the two groups ( $27.27 \%$ vs $22.64 \%, p=0.389$ ), the in-hospital length of stay, as well as ICU length of stay, was significantly shorter in the experimental group (11.88 \pm 1.469 vs $16.42 \pm 1.290$, $p=0.026 ; 7.545 \pm 0.866$ vs $10.11 \pm 0.793, p=0.038$ ). Meanwhile, the MODS incidence was also similar $(51.52 \%$ vs $49.06 \%, p=0.064)$, which suggests that higher CK levels would not predict the appearance of MODS. Table 4. 
Outcomes of subjects with RM associated AKI after CRRT termination.

\begin{tabular}{|lllll|}
\hline Outcomes & $\begin{array}{l}\text { All } \\
(\mathbf{n = 8 6})\end{array}$ & $\begin{array}{l}\text { CK }>\mathbf{5 0 0 0} \text { U/L } \\
(\mathbf{n}=\mathbf{3 3})\end{array}$ & $\begin{array}{l}\text { CK<5000 U/L } \\
(\mathbf{n}=\mathbf{5 3})\end{array}$ & P value \\
\hline Length of CRRT periods & $6.698 \pm 0.850$ & $4.879 \pm 0.792$ & $7.830 \pm 0.909$ & $\mathbf{0 . 0 2 7}$ \\
\hline In-hospital Mortality & $21(24.42)$ & $9(27.27)$ & $12(22.64)$ & 0.389 \\
\hline In-hospital Stay Length & $14.67 \pm 1.380$ & $11.88 \pm 1.469$ & $16.42 \pm 1.290$ & $\mathbf{0 . 0 2 6}$ \\
\hline ICU Stay Length & $9.128 \pm 0.830$ & $7.545 \pm 0.866$ & $10.11 \pm 0.793$ & $\mathbf{0 . 0 3 8}$ \\
\hline MODS Incidence & $43(50.00)$ & $17(51.52)$ & $26(49.06)$ & 0.064 \\
\hline $\begin{array}{l}\text { CK: Creatine Kinase; CRRT: Continuous Renal Replacement Therapy; ICU : Intensive Care Unit; MODS: } \\
\text { Multiple Organ Dysfunction Syndrome. }\end{array}$ & & & \\
\hline
\end{tabular}

\section{Outcomes of subgroups with CK $>5,000 \mathrm{U} / \mathrm{L}$ after CRRT termination}

For thirty-three patients with CK above 5,000 U/L after CRRT termination, there were 13 cases with CK > $15,000 \mathrm{U} / \mathrm{L}$, with the remaining cases within 5,000 - 15,000 U/L. These participants presented a similar length of CRRT period $(p=0.079)$, as well as in-hospital mortality $(p=0.579)$. In addition, the in-hospital and ICU length of stay were also comparable between these two subgroups ( $p=0.086, p=0.099)$. Finally, there was no higher incidence of MODS for patients with severe RM after CRRT termination $(p=0.090)$. Table 5.

Table 5

Outcomes of subgroups with $\mathrm{CK}>5000 \mathrm{U} / \mathrm{L}$ after CRRT termination.

\begin{tabular}{|llll|}
\hline Outcomes & $\begin{array}{l}\text { CK }>15000 \mathrm{U} / \mathrm{L} \\
(\mathrm{n}=13)\end{array}$ & $\begin{array}{l}\text { CK : 5000 }-15000 \mathrm{U} / \mathrm{L} \\
(\mathrm{n}=\mathbf{2 0})\end{array}$ & P value \\
\hline Length of CRRT periods & $3.154 \pm 0.741$ & $6.000 \pm 1.161$ & 0.079 \\
\hline In-hospital Mortality & $5(38.46)$ & $4(20.00)$ & 0.579 \\
\hline In-hospital Stay Length & $8.923 \pm 1.542$ & $12.80 \pm 1.445$ & 0.086 \\
\hline ICU Stay Length & $5.769 \pm 1.340$ & $8.700 \pm 1.081$ & 0.099 \\
\hline MODS Incidence & $7(53.85)$ & $10(50.00)$ & 0.090 \\
\hline $\begin{array}{l}\text { CK: Creatine Kinase; CRRT: Continuous Renal Replacement Therapy; ICU : Intensive Care Unit; MODS: } \\
\text { Multiple Organ Dysfunction Syndrome. }\end{array}$ & & \\
\hline
\end{tabular}




\section{Correlations Between Ck And Other Variables After Crrt Termination}

Since CK levels were significantly higher in the experimental group, we performed a linear correlation between CK and other variables after CRRT termination. The results of this study suggest a positive correlation between CK and myoglobin $(r=0.3534, P=0.0020)$. For the renal function markers, such as urine output, creatinine, urea nitrogen, and eGFR, none of the above factors were associated with CK levels (all $p$-value $>0.05$ ). Figure 3 .

\section{Discussion}

$\mathrm{RM}$ is a syndrome characterized by rapid breakdown and leakage of skeletal muscle cell contents[19], such as myoglobin, CK, and other cytokines. During the past several decades, there have been multiple and diverse causes of RM, which include trauma, toxins, infections, drugs, and strenuous exercise[2026]. The major cause of RM in our study was determined to be infections (36.2\%), including Staphylococcus aureus, Streptococcus pneumoniae, and klebsiella pneumoniae.. All 86 cases presented with certain classical symptoms, such as muscle weakness, myalgia, or dark - colored urine, along with significantly higher CK levels, and without red blood cells in the urine sediment.

Among the various forms of $\mathrm{RM}$, the most common pathophysiologic feature is attributed to a rise in intracellular ionized calcium from the injury muscles, which brings a loss of the transcellular calcium gradient, and this eventually leads to cell death [11]. The result of these cellular impairments is the release of $\mathrm{CK}$, myoglobin, and various electrolytes into the blood circulation, and this results in a classic clinical presentation, as well as RM - induced AKI. RM itself makes a fluid sequestration, which decreases renal perfusion, activates the Renin-Angiotensin -Aldosterone System (RAAS), and results in renal vasoconstriction[3]. In addition, RM-associated AKI is believed to be triggered by myoglobin, which in this context is considered to be a toxin that causes renal dysfunction. Myoglobin could potentially precipitate with Tamm-Horsfall protein, which could lead to the tubular obstruction. Additionally, the heme moiety released from myoglobin could promote free radical formation, which accelerates direct and ischemic tubular injury. All of the above potentially contribute to RM - induced AKI[5].

According to the RIFLE classification system, all participants in our study belonged to the "Risk" "Injury" or "Failure" stages of renal function. In order to prevent the deterioration of RM-associated AKI, traditional fluid resuscitation, including crystal and colloid liquid, was applied for all patients. For those with remarkable metabolic acidosis, sodium bicarbonate therapy was used to stabilize the homeostasis[27]. Additionally, CRRT was chosen because all patients with RM-associated AKI met at least one of the intervention criteria. 
Data from several previous studies have identified the effect of CRRT on RM- associated AKI. As early as 2012, Zhang Ling et.al showed that CVVH could be employed to clear myoglobin effectively in patients with RM - induced AKI and presented oliguria[10]. After that, Wangxin Tang et.al (2013) confirmed the renal protective effects of CVVH in a glycerol - injection - induced RM model; specifically they found that CVVH could improve mitochondrial function and reduce tubular epithelial apoptosis through a dose effect relationship[13]. In addition, there have been an increasing number of reports on High Cut-Off (HCO) hemodiailtration for removal of myoglobin in RM-associated AKI. Research published by Nils Heyne et al (2012) concluded that HCO CRRT was a highly effective means for elimination of myoglobin and a novel therapeutic approach to severe RM - induced AKI[8]. Similarly, Vladimir Premru et al (2013) also supported the use of HCO hemodiafiltration for rapid initial removal of myoglobin in severe acute myoglobinuric kidney injury[28]. In recent years, several case reports have investigated the effect of plasma exchange, as well as plasma filtration adsorption, on RM-associated AKI, and proved a successful reduction of myoglobin levels, along with preservation of renal function[11, 12, 29]. Participants in our study, had a significantly high McMahon score, as well as worse renal function on admission, which suggested a necessity for renal replacement therapy. Despite the fact that CRRT is reputedly safe and efficacious for $\mathrm{RM}$ - induced $\mathrm{AKI}$, questions have still been raised regarding its use.

According to the British Renal Association guidelines about AKI, if the urine output is $>1,000 \mathrm{~mL} / \mathrm{D}$, and serum creatinine $\leq 265 \mathrm{umol} / \mathrm{L}$, CRRT could be considered for suspension [18]. However, for RMassociated AKI, whether CK levels should be taken into account for CRRT termination, seems to be a common problem for therapy. For one, moderate RM with CK above 5,000 U/L, predicts an increased risk of renal injury. Additionally, severe RM, which CK > 15,000 U/L, shows a higher risk of dialysis. Further, patients with a higher level of CK suggests a potential ongoing muscle injury or incomplete recovery after treatments.

Two retrospective reviews of 30 and 41 cases about exertional RM-associated AKI, found discharged CK values ranging from 1,410 to $94,665 \mathrm{U} / \mathrm{L}$ and 10 to $61,617 \mathrm{U} / \mathrm{L}[30,31]$. Mario Pezzi et.al (2017) reported case series about the use of coupled plasma filtration adsorption (CPFA) in traumatic RM, and the lowest CK level was 9,000 U/L, and the highest level was 20,000 U/L when CPFA stopped[11]. Olcay Dilken et.al (2019) presented a successful reduction of CK levels to 40,000 U/L in severe RM using extracorporeal blood purification (CytoSorb) after termination[12]. Recently, an article published by Eka Laksmi Hidayati et al (2020) displayed a progressive reduction of CK levels to $61 \mathrm{U} / \mathrm{L}$ for multiple wasp stings-induced RM through continuous plasma exchange[29]. To our knowledge, there is still no consensus on the confirmed CK levels that should be reduced by CRRT.

In our study, we investigated whether CK levels should also be considered in CRRT therapy for patients with RM-associated AKI. Patients were divided into two groups, and those with CK > 5,000 U/L after termination were assigned to the experimental group. Laboratory examinations, such as routine blood tests, CK levels, myoglobin, renal function indicators, and inflammatory biomarkers, were comparable between the two groups on admission. After CRRT termination, the APACHEll score, as well as McMahon score, revealed differences. The average McMahon score for all patients was below six, which predicted a 
lower requirement for renal replacement therapy again[3]. The result of our study also found that serum myoglobin was significantly higher in the experimental group, except for an obvious high level of CK. The renal function indicators, such as urine output, creatinine, urea nitrogen, and eGFR, along with inflammatory biomarkers, were consistent between patients after CRRT. Although in-hospital mortality showed no difference, the slength of stay for in-hospital, as well as ICU, was significantly lower, and the CRRT period was significantly shorter for the higher CK level group. Another important finding was a positive correlation between CK and myoglobin after CRRT treatment, but there was no evidence that CK had any relationship to renal function indicators.

To the best of our knowledge, there is an accumulation of CK and myoglobin in the blood after muscle damage. CK reaches its maximum value after around 24 hours, and then is eliminated by oxidation in the blood; this process is independent from liver and kidney function. Myoglobin reaches its maximum value within $12 \mathrm{~h}$ and is rapidly cleaned up by the kidneys, if kidney function is unrestricted[1] CK and myoglobin are sensitive indicators for striated muscle injury. For patients with RM-associated AKI, a large amount of myoglobin is released into the blood, which exceeds renal excretion capacities and causes nephrotoxic effects. In this case, the levels of CK and myoglobin may show a consistent exponential growth with respect to each other, along with persistent muscle damage. CRRT, an approach to aggressively remove the serum uremia-related molecules (such as creatinine and urea nitrogen), mainly aims to protect renal function, while its effect on sustained muscle injury is limited[32]. Our study found that there was no relationship between CK and renal function markers after CRRT by linear correlation analysis.

Lastly, we performed a subgroup analysis for patients with $\mathrm{CK}>5,000 \mathrm{U} / \mathrm{L}$, since it included those with severe RM at the end of CRRT. It is interesting to note that neither in-hospital mortality nor length of stay showed any difference among subgroups. These results suggest that if renal functions are significantly improved by CRRT, CK levels could be gradually returned to normal through traditional supportive treatments, such as fluid resuscitation and alkalization of urine. Importantly, on the premise of improved renal functions, a higher CK level might not be an independent risk factor for in-hospital mortality.

There were several potential limitations associated with the research presented here that need to be highlighted. First, our study only divided patients into two groups according to CK levels after CRRT termination. We could not identify an appropriate range that CK should be decreased to that would guarantee low recurrence of RM - induced AKI. Second, since there was a positive correlation between CK and myoglobin at the end of CRRT, it is unlcear whether high levels of myoglobin would lead to a poor prognosis, and this requires further investigation.. Lastly, as this was a single center and retrospective analysis, selection bias cannot be ruled out. In addition, with the development of new and more advanced therapeutics over the past decade, relative biases cannot be ruled out. It is possible that a larger, randomized and controlled trial over an extended period of time could help to override these limitations.

\section{Conclusion}


For patients with rhabdomyolysis-associated acute kidney injury, CRRT may be independent of CK levels, if renal functions have recovered to an appropriate level. Prospective clinical trials would be needed to investigate and confirm the optimal CK range that could be used as a guide to help prevent the recurrence of renal impairments after treatments.

\section{Declarations}

\section{Acknowledgements}

The authors would like to thank the authors of all the published research that contributed to the data used in this study.

\section{Authors' contributions}

Conceptualization: LX, XR. Data acquisition: LX, XR. Data analysis: XR, YZ, YL. Data interpretation: YZ, YL. Manuscript preparation: LX, XR, SL. Manuscript revision: all authors. All authors read and approved the fnal manuscript.

\section{Funding}

This research was supported by the Development Scheme of Tongji Hospital Affiliated to Tongji Medical College of Huazhong University of Science and Technology, Wuhan, China, Grant 2020YFC0843701. The funding body played a role in the design of the study and collection, analysis, interpretation of data, and in preparation for manuscript.

\section{Availability of data and materials}

The datasets used and/or analysed during the current study are available from the corresponding author on reasonable request.

\section{Ethics approval and consent to participate}

This study was approved by the Human Research Ethics Committee of the Tongji Hospital, written informed consent was obtained from all participants. We confirmed that all methods were performed in accordance with the relevant guidelines and regulations of Declaration of Helsinki.

\section{Consent for publication}

Not consent is required.

\section{Competing interests}

None of the authors declare that there were any competing interests.

\section{Author details}


${ }^{1}$ Tongji Medical College of Huazhonng University of Science and Technology, Wuhan 430030, China;

${ }^{2}$ Department of Intensive Care Unit of Tongji Hospital Affiliated to Tongji Medical College of Huazhong University of Science and Technology, Wuhan 430030, China

\section{References}

1. Scharf C, Liebchen U, Paal M, Irlbeck M, Zoller M, Schroeder I. Blood purification with a cytokine adsorber for the elimination of myoglobin in critically ill patients with severe rhabdomyolysis. Crit Care 2021;25.

2. Zeng X ZLWT. Continuous renal replacement therapy (CRRT) for rhabdomyolysis (Review). Cochrane Db Syst Rev 2014.

3. Cabral BMI, Edding SN, Portocarrero JP, Lerma EV. Rhabdomyolysis. Disease-a-Month 2020;66:101015.

4. Simpson JP, Taylor A, Sudhan N, Menon DK, Lavinio A. Rhabdomyolysis and acute kidney injury: creatine kinase as a prognostic marker and validation of the McMahon Score in a 10-year cohort. Eur J Anaesth 2016;33:906-912.

5. Petejova N, Martinek A. Acute kidney injury due to rhabdomyolysis and renal replacement therapy: a critical review. Crit Care 2014;18:224.

6. Mcmahon GM, Zeng X, Waikar SS. A Risk Prediction Score for Kidney Failure or Mortality in Rhabdomyolysis. Jama Intern Med 2013;173:1821.

7. Weidhase L, de Fallois J, Haußig E, Kaiser T, Mende M, Petros S. Myoglobin clearance with continuous veno-venous hemodialysis using high cutoff dialyzer versus continuous veno-venous hemodiafiltration using high-flux dialyzer: a prospective randomized controlled trial. Crit Care 2020;24.

8. Heyne N, Guthoff M, Krieger J, Haap M, Häring H. High Cut-Off Renal Replacement Therapy for Removal of Myoglobin in Severe Rhabdomyolysis and Acute Kidney Injury: A Case Series. Nephron 2013;121:c159-c164.

9. Rodríguez E, Soler MJ, Rap O, Barrios C, Orfila MA, Pascual J, et al. Risk factors for acute kidney injury in severe rhabdomyolysis. Plos One 2013;8:e82992-e82992.

10. Zhang L, Kang Y, Fu P, Cao Y, Shi Y, Liu F, et al. Myoglobin clearance by continuous venous-venous haemofiltration in rhabdomyolysis with acute kidney injury: A case series. Injury 2012;43:619-623.

11. Pezzi M, Renda S, Giglio AM, Scozzafava AM, Tiburzi SP, Casella P, et al. The Use of Coupled Plasma Filtration Adsorption in Traumatic Rhabdomyolysis. Case Reports in Critical Care 2017;2017:1-8.

12. Dilken $O$, Ince $C$, van der Hoven B, Thijsse S, Ormskerk P, de Geus HRH. Successful Reduction of Creatine Kinase and Myoglobin Levels in Severe Rhabdomyolysis Using Extracorporeal Blood Purification (CytoSorb®). Blood Purificat 2020;49:743-747.

13. Wangxin Tang ZCWW. Renal Protective Effects of Early Continuous VenovenousHemofiltration in Rhabdomyolysis. Artif Organs 2013;4:390-400. 
14. Hui C, Shen-Ying W, Chao L. Value of hemoperfusion combined with continuous renal replacement therapy in rhabdomyolysis complicated with acute renal injury in-duced by bee stings. Journal of Clinical and Experimental Medicine 2020;19:1764-1767.

15. Li B, Wang X, Li C. Curative Effectiveness Observation of Early Sequential Hemoperfusion Combined with Glucocorticoid on Acute $\mathrm{I}^{1} / 4^{2}$ habdomyolysis with Bee Stings. HEBEI MEDICINE 2020;26:15511556.

16. Li Chang-Sheng YYCL. Clinical evaluation of early sequential hemoperfusion in treatment of rhabdomyolysis by acute bee stings. Chinese J Ind Med 2018;5:327-330.

17. Dezheng $\mathrm{C}$, Zhang L, Li M, Zhang Y, Fang H, Xu W, et al. Continuous renal replacement therapy for rhabdomyolysis with acute kidney injury following multiple wasp stings. West China Medical Journal 2018;7:848-851.

18. Nye NS, Kasper K, Madsen CM, Szczepanik M, Covey CJ, Oh R, et al. Clinical Practice Guidelines for Exertional Rhabdomyolysis: A Military Medicine Perspective. Curr Sports Med Rep 2021;20:169-178.

19. Park Y, Song JY, Kim SY, Kim SH. Clinical Characteristics of Rhabdomyolysis in Children: Single Center Experience. Childhood Kidney Diseases 2018;22:52-57.

20. Secombe P, Milne C. Hyponatraemia-induced rhabdomyolysis complicated by anuric acute kidney injury: a renal replacement conundrum. BMJ Case Reports 2016:bcr2016218198.

21. Kovid Trivedi RH. Role of Plasma Exhange With Continuous Renal Replacement Therpay (CRRT) for Rhabdomyolysis and Associated Renal Failure Secondary to synthetic Cannabinoid Abuse. Chest Annual Meeting 2017.

22. Ahmad A, Zain MA, Ashfaq AA, Ullah W. A Late Presentation of Substance-related Rhabdomyolysis with Normal Serum Creatine Kinase Levels and Complicated with Acute Tubular Necrosis. Cureus 2019;3:1-5.

23. Wiegele M, Krenn CG. Cytosorb ${ }^{\text {TM }}$ in a Patient with Legionella Pneumonia-Associated Rhabdomyolysis. Asaio J 2015;61:e14-e16.

24. Luckoor P, Salehi M, Kunadu A. Exceptionally High Creatine Kinase (CK) Levels in Multicausal and Complicated Rhabdomyolysis: A Case Report. American Journal of Case Reports 2017;18:746-749.

25. Al Badi A, Al Rasbi S, Alalawi AM. Exercise-Induced Rhabdomyolysis: A Case Report and Literature Review. Cureus 2020;8:1-7.

26. Sunder A, Mohanty B, Singh A, Yadav P. Rhabdomyolysis - Exercise induced nightmare. Journal of Family Medicine and Primary Care 2019;8:305.

27. Nielsen JS, Sally M, Mullins RJ, Slater M, Groat T, Gao X, et al. Bicarbonate and mannitol treatment for traumatic rhabdomyolysis revisited. The American Journal of Surgery 2017;213:73-79.

28. Premru V, Kovač J, Buturović-Ponikvar J, Ponikvar R. Some Kinetic Considerations in High Cut-Off Hemodiafiltration for Acute Myoglobinuric Renal Failure. Ther Apher Dial 2013;17:396-401.

29. Hidayati EL, Rosyady M, Puspitasari HA, Saraswati M, Grace A, Ambarsari CG. Plasma exchange and hemodialysis for severe manifestations of multiple wasp stings in a child. Journal of renal injury 
prevention 2020;9:e27-e27.

30. Arnautovic JZ, Tereziu S. Evaluation of Clinical Outcomes in Hospitalized Patients With Exertional Rhabdomyolysis. The Journal of the American Osteopathic Association 2019;119:428.

31. Oh RC, Arter JL, Tiglao SM, Larson SL. Exertional Rhabdomyolysis: A Case Series of 30 Hospitalized Patients. Mil Med 2015;180:201-207.

32. Masakane I, Sakurai K. Current approaches to middle molecule removal: room for innovation. Nephrol Dial Transpl 2018;33:iii12-iii21.

\section{Figures}

\section{Figure 1}

Laboratory examinations on admission.

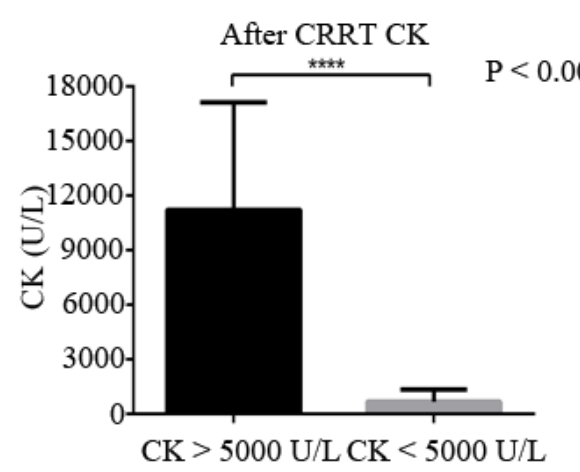

After CRRT Creatinine

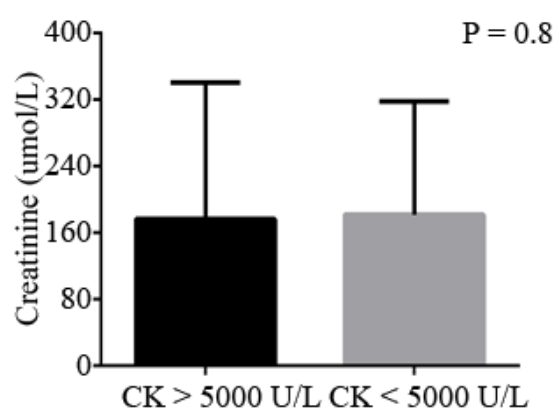

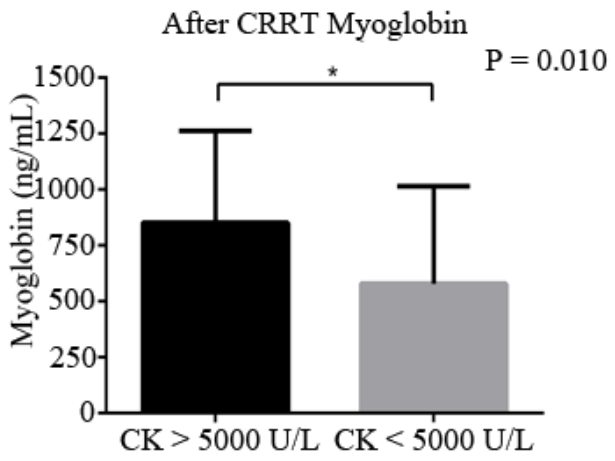

After CRRT Urea Nitrogen

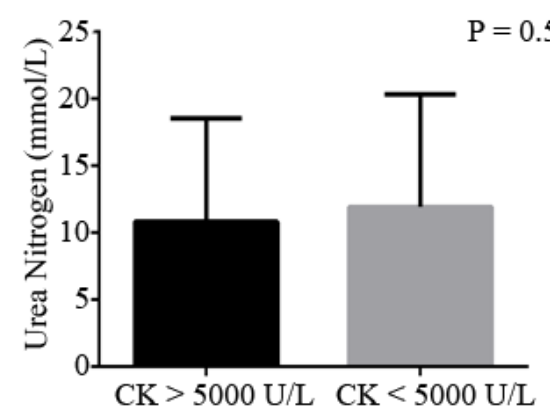

After CRRT Urine Output

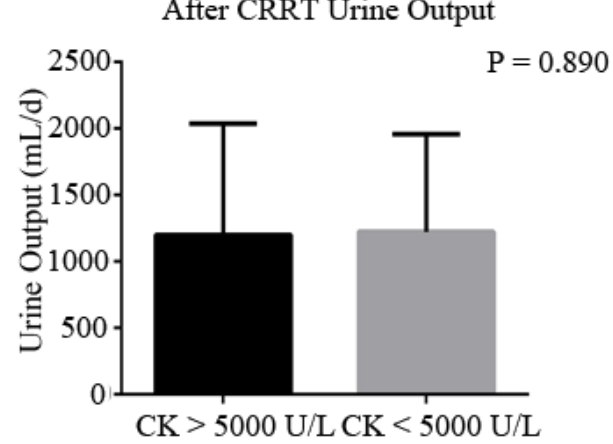

After CRRT eGFR

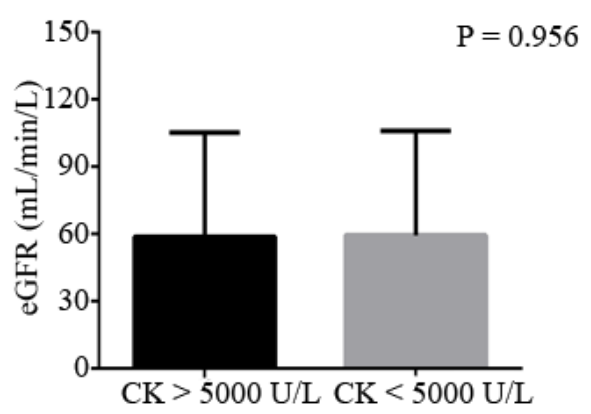

\section{Figure 2}

Laboratory Examinations after CRRT termination. 

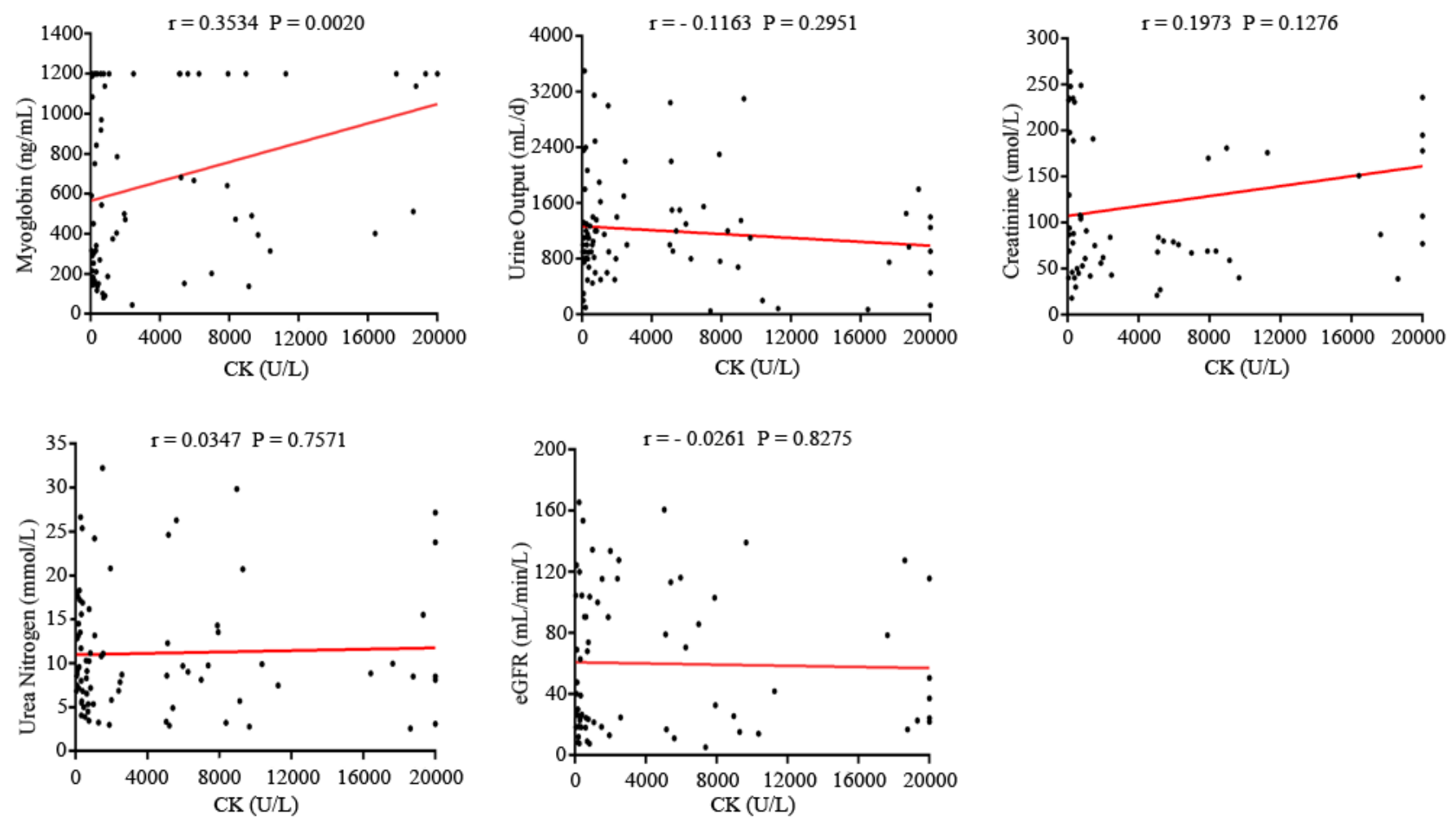

Figure 3

Correlation between CK and Myoglobin,Urine Output, Creatinine, Urea Nitrogen, eGFR. 